\title{
FACTORS CONTRIBUTING TO DELAYED AMBULANCE RESPONSE TIME USING AMBULANCE VEHICLE LOCATOR AND GLOBAL INTEGRATING SYSTEM (AVL- GIS) AT HOSPITAL UNIVERSITI SAINS MALAYSIA
}

\author{
Zulaili Asri ${ }^{1}$, Mohd Boniami Yazid ${ }^{1,2}$, Mohd Shaharudin Shah Che Hamzah ${ }^{1}$, Normalinda Yaacob ${ }^{1}$, Tuan \\ Hairulnizam Tuan Kamauzaman ${ }^{1}$ and Nik Hisamuddin Nik Ab. Rahman ${ }^{1,2}$ \\ ${ }^{1}$ Department of Emergency Medicine, School of Medical Science, Universiti Sains Malaysia Health Campus, \\ 16150 Kubang Kerian, Kelantan, Malaysia \\ ${ }^{2}$ Hospital Universiti Sains Malaysia, 16150, Kubang Kerian, Kelantan, Malaysia.
}

Corresponding author: Mohd Boniami Bin Yazid

Email: boniami@usm.my

\begin{abstract}
This study aims to determine ambulance response time in Kubang Kerian district, Kelantan, Malaysia using Ambulance Vehicle Locator and Global Integrating System (AVL-GIS) device and the factors associated with delayed response time. This was a 6-months prospective, cross-sectional study conducted at Emergency and Trauma Department, Hospital Universiti Sains Malaysia (HUSM) in Kubang Kerian, Kelantan, Malaysia. The possible factors identified to have caused delayed ambulance response time from previous literature were included in this study. All prehospital cases from September 2017 until February 2018 attended to by AVL-GIS installed ambulances were included in this study. Data were obtained from the standardized prehospital Proforma forms and extracted from AVL-GIS using iGPS software. Simple and multiple logistic regression tests were used to determine the factors associated to delayed response time. From 505 cases, $75 \%$ found to be delayed case meanwhile 25\% is non delayed case as defined in study protocol. Further analysis on delayed cases show that call duration, caller type, traffic condition, travelling time and team allocation time were associated with delayed ambulance response time. Evaluation of each component contributing to delayed ambulance response time is needed for intervention to improve prehospital care in the future.
\end{abstract}

Keywords: ambulance, AVL-GIS, emergency medical service, prehospital, response time

\section{INTRODUCTION}

Ambulance response time is the duration taken from receiving an emergency call in the call centre until an ambulance arrives at the scene. Since 1979, Emergency Medical Services (EMS) in the United States had adopted 8 minutes ambulance response time as international standard because of higher survival rates in patients with cardiac arrest who received earlier advanced life support $(A L S)^{1}$. Many international and local studies had been conducted to improve ambulance response time as it has been accepted as one of the indicators for stratifying the quality of prehospital care in terms of service and patient's satisfaction ${ }^{3-}$ 9 .

This study is conducted in the district of Kubang Kerian, Kelantan, Malaysia with a population size of 58,000 within $20.1 \mathrm{~km}^{2}$ area ${ }^{10}$. The ambulance service is based in Hospital Universiti Sains Malaysia (HUSM), a tertiary teaching hospital with 800 beds. Ambulance response time in the said district is determine using Ambulance Vehicle Locator and Global Integrating System (AVL-GIS) device that been installed in the dedicated ambulance.

In this study, AVL-GIS was used to estimate the time and distance of ambulance travel to the scene. We chose this method considering the fact that based on a previous study, the mean travelling time calculated manually was not very accurate, in that it was overpredicted in urban area and underpredicted in suburban and rural area compared to the mean time using AVL-GIS ${ }^{7}$.

In short, the main objective of our study is to determine the mean ambulance response time, while our specific objective is to study the association between both internal and external factors that had been identified from previous literature towards delayed ambulance response time in the district. High call volume, availability of crew and ambulance, location of scene, weather, traffic condition, distance and geographical factors are common factors associated with delayed ambulance response time in all of the related studies ${ }^{2-6,11}$.

Hence, this study would add further information and may be used as guidance for initiating interventional measures to improve ambulance response time and prehospital care in the district as well as other area. 


\section{METHODS}

This was a prospective cross-sectional study conducted for a 6 months period from $1^{\text {st }}$ September 2017 to $28^{\text {th }}$ February 2018 at Hospital Universiti Sains Malaysia (HUSM), Kubang Kerian, Kelantan, Malaysia. The data were obtained from two methods and integrated in Statistical Package for Social Sciences (SPSS) version 22.0 software. First method was data obtained from the standardized Profoma forms which were filled up by the Emergency Medical Service (EMS) personnel on duty. The form consists of demographic data of the call taker, caller, details of the responded case, all the recorded time including time of call received, team allocation, ambulance arrival and patient contact and details during the journey of ambulance. Second method of data collection was from the device installed within the ambulance. Our ambulances were installed with Ambulance Vehicle Locator and Global Integrating System (AVL-GIS) device (Model: Skyline-V3/V4) in which it would automatically record the ambulance coordinates, travel distance, and time of departure and arrival to the scene for each case that responded by prehospital team. All this information can be assessed by the investigator through igps software.

Sample size calculation was referred to Peduzzi et al $(1996)^{12}$, based on objective of this study and number of events per variable (EPV) as included in analysis table. Convenience sampling method is use as all the response case by our Emergency Medical Service within the study period are include in this study

The ambulance response time was calculated as a sum of the call duration plus team allocation time plus travelling time until ambulance reach the scene by using AVL- GIS records. We were using 8 minutes as a benchmark for ambulance response time based on international standard. The cases included in this study were then divided into 2

\section{RESULTS}

A total of 505 ambulance calls during this 6 months' period were included in this study. From this number, 376 cases $(74.5 \%)$ belong to the 'delayed group' and 129 cases (25.5\%) belong to the 'non-delayed group'. The mean response time is $\mathbf{1 2 . 4 2}$ minutes. Descriptive analysis for each factor is listed in Table 1. Fraction of each component of the ambulance response time is described in Table 2 . Travelling time contributes to $64 \%$ of total ambulance response time followed by team activation time.

Table 3 shows the relationship between all internal factors towards delayed ambulance response time. Factors identified to be significantly associated with delayed response time are caller type and call duration $(p<0.05)$. groups: (i) delayed (response time more than 8 minutes. (ii) non-delayed (response time 8 minutes and less).

Factors associated with delayed ambulance response time were divided into internal factors and external factors. Internal factors are factors that are related to emergency calls received in call centre including the caller type, call receiver and call duration. External factors are other aspects following the calls, starting from team activation until the ambulance reached the scene, that can contribute to the delayed response time such as weather, road condition, traffic condition, duration of team activation and ambulance journey to the incident site.

The data was analysed with SPSS version 22.0. We reported the descriptive analysis as frequency, mean and standard deviation of demographic data. For statistical analysis, the dependent variables were identified as delayed and nondelayed groups based on definition during research protocol stage. Meanwhile, the independent variables are the various factors that can cause delayed response time which were further divided into external and internal factors. Univariate analysis by simple logistic regression test was used to look for relationship between each factor included in the study towards the occurrence of delayed ambulance response time. From the results given, we took the variables which were significant or biologically plausible to be included, even though they were not significant in univariate analysis, to proceed with multivariate analysis. The dependent variables in our study are the occurrence of delayed or nondelayed ambulance response time which are binominal outcomes. Hence, multiple logistic regression test was used to study the association between binominal categorical dependent variables (delayed or not delayed) and multiple independent variables (factors cause delayed response time).

The results obtained from simple logistic regression test showed that for a unit increase in call duration, there is $67 \%$ higher probability to experience delayed ambulance response time (OR: $1.67,95 \% \mathrm{Cl}: 1.37,2.05)$. In contrast to background of call receiver, the caller type was associated with delayed response time in which the public had higher probability for delayed response time when they make the emergency call to call centre in comparison with MECC and other callers. It was reported that MECC showed $38 \%$ odd to delayed response time compared to public $(\mathrm{p}<0.001, \mathrm{OR}=0.38,95 \% \mathrm{Cl}: 0.24,0.59)$, whereas for other callers there were $16 \%$ odd to delayed response time compared to public when they make the calls $(p<0.001, O R=0.16,95 \% \mathrm{Cl}$ : $0.07,0.34)$. The significant internal factors associated with delayed ambulance response time $(p<0.05)$ are caller type and call duration. 
Relationship of external factors towards delayed ambulance response time is shown in Table 4. Simple logistic regression test was used for this univariate analysis and it was found that team allocation time, travelling time, traffic condition and ambulance mechanism used were the only external factors that significantly related to occurrence of delayed ambulance response time $(p<0.05)$.

The duration of time from team activation until ambulance team reached the scene was significantly associated with delayed ambulance response time. This includes the team's allocation time and travelling time of ambulance. The result

For the response location, the weather and time during the ambulance call were not significantly associated with delayed ambulance response time. Multiple logistic regression test was used for multivariate analysis between both internal and external factors towards delayed ambulance response time. As shown in Table 5, using forward method in logistic regression test, we found that the team allocation time and travelling time were the only factors that are significantly associated

\section{DISCUSSION}

Previous studies conducted in the same setting at Hospital Universiti Sains Malaysia, Kubang Kerian, Kelantan, Malaysia in 2008 showed that mean ambulance response time was 15 minutes $^{2}$. After 10 years, the response time improved to 12 minutes in average which is still far from international standard. Using 8 minutes as the benchmark of ambulance response time, there was $75 \%$ cases of delayed response time from a total of 505 cases within the 6 months period of study.

Numerous factors from the moment the call centre received a call until the ambulance reach the response location had been considered in this study in which it was further divided into internal factors and external factors. From the analytical statistics, few factors were found to be significantly associated with delayed ambulance response time in our setting at Hospital Universiti Sains Malaysia, Kubang Kerian, Kelantan, Malaysia. For internal factors, the call duration and caller type are associated with delayed response time. This is similar to the study conducted in Singapore which found out that high call volume was predicted to cause prolonged response time ${ }^{3}$. Mean time for call duration was 2.11 minutes which can be prolonged resulting from caller factor or call receiver factor. For the caller, anyone in public who makes an emergency call has the tendency to cause delayed response time as compared to a call made by MECC or others which referred to other emergency sectors, clinic or private EMS within the coverage of the ambulance service. Due to lack of medical awareness and familiarity of surrounding among shows that a unit increase in team allocation time means 1.5 times higher probability to delay ambulance response time $(p<0.001, O R=1.57$, 95\% Cl: 2.82, 4.85) and a unit increase in travelling time will result in 3.7 times higher probability to delay ambulance response time $(p<0.001, O R=3.7,95 \% \mathrm{Cl}: 1.32,1.85)$. For heavy traffic, it was reported that there were $47 \%$ odd to delayed cases compared to non-heavy $(\mathrm{p}<0.001, \mathrm{OR}=0.47,95 \% \mathrm{Cl}: 0.25,0.90)$. In a case of delayed response, there is 3 times higher probability the ambulance will use light and siren compared to siren only $(p<0.001, O R=2.88,95 \%$ $\mathrm{Cl}: 1.80,4.61)$. Other ambulance mechanism was not significantly used in delayed response case.

towards delayed ambulance response time $(p<0.001)$. For a unit increase in travelling time (GIS), there was 6.33 times to have delayed response time $(95 \% \mathrm{Cl}: 4.06,9.88)$. For a unit increase in team allocation time, there was 4.41 times to have delayed response time $(95 \% \mathrm{Cl}$ : $2.73,7.12)$. There was no significant association between other factors tested towards delayed ambulance response time from this regression test.

the public, the call receiver might have difficulty to obtain the information regarding the type of emergency and location from them, which will lead to longer call duration in comparison with EMS and the staff working in call centre who been trained on answering call using provided guideline.

In our study, the external factors which were significantly associated with delayed response time were team allocation time, travelling time, traffic condition and ambulance mechanism used. Time for team allocation starts from the point of team activation until ambulance depart from base. The availability of the crew for prehospital care was identified as one of the factors that can affect the response time in Ireland ${ }^{4}$. In our setting, once the call receiver ended the call, the EMS team will activate the alarm to alert the person who was in charge of prehospital service during that shift before they depart to the scene. Time will be delayed if the person in the team is in the clinical area at the same time and took longer time to be in the ambulance. Hence, in future there should be an allocation for prehospital care team among medical officers and paramedics trained in EMS who are not involved in the clinical area.

Travelling time in this study was positively correlated with the distance of ambulance travel. We used travelling time measured by AVL-GIS because it is more accurate than measuring manually in which it took 1.31 minutes longer for the latter.

The road condition in Kubang Kerian and also other area of coverage for our prehospital care 
are considered sub-urban and most of the roads are paved, lack of alternative or shortcut way. In this case, the road condition was not the main reason for delayed travelling time. Bad road condition increases the travelling time in which the incident scenes were far from the main road, unclear location was given, wrong addresses were given and narrow roads condition on the way to the scene. The choice of reasons of delayed ambulance arrival to the scene have been provided in the Proforma form which were identified by the respective EMS personnel in the ambulance.

The traffic condition is a well-known factor in most of the studies on delayed response time ${ }^{5-}$ 7. Heavy traffic condition observed in this study such as congested road during peak hour and lack of cooperation from public affected the speed of ambulance travel and caused the delay in response time.

Other external factors studied were not significantly associated but important to be highlighted such as the weather, scene location, call triage and time of response. The weather was not a significant factor of delayed response time in this study which is different from the result obtained in a study conducted in Singapore. The weather will affect the view of the road during rainy season, slippery roads which causes the ambulance to reduce its speed while responding to case $^{6}$. As our study was conducted outside of monsoon time, most of the response time occurred during good weather. Response location was identified as one of the risk factors from previous literature as there will be delayed response time when the scene is inside a building. Vertical barrier will impede the movement of paramedics to patient and prolong the time interval ${ }^{8}$.

Another study conducted found that the higher the building, the more time is needed to gain access to the patient and the more delay it cause in response time?. However, in our study it was not the case as there is no high rise building within the coverage area and there is road access for most of the case responded.

Table 1: Descriptive analysis of each factors (internal and external)

\begin{tabular}{lll}
\hline Factors & Mean (SD) & N (\%) \\
\hline Internal Factors & & \\
Patient's Age & $40.50(9.81)$ & \\
Patient's Gender & \\
Male & $321(63.5)$ \\
Female & $148(29.3)$ \\
Call receiver* & \\
EMD & & $411(81.4)$ \\
AMO/SN & $34(6.7)$ \\
APM & $58(11.5)$ \\
Working Experience (years) & $11.19(6.42)$ \\
Highest Academic Qualification ${ }^{\mathfrak{E}}$ & & \\
SPM/STPM & & $432(85.5)$ \\
Diploma & $29(5.7)$ \\
Degree & & $9(1.8)$ \\
Call processing time (minutes) & \\
Team allocation time (minutes) & $2.11(1.24)$ \\
\hline
\end{tabular}


Table 1: Continue

\begin{tabular}{|c|c|c|}
\hline Demographic & Mean (SD) & N (\%) \\
\hline \multicolumn{3}{|l|}{ External Factors } \\
\hline \multicolumn{3}{|l|}{ Traffic condition } \\
\hline Heavy & & $79(15.6)$ \\
\hline Non heavy & & $424(84.0)$ \\
\hline \multicolumn{3}{|l|}{ Weather } \\
\hline Good weather & & 454 (89.9) \\
\hline Bad weather & & $51(10.1)$ \\
\hline \multicolumn{3}{|l|}{ Place of incident } \\
\hline Outside building & & $180(35.6)$ \\
\hline Inside Building & & $324(64.2)$ \\
\hline \multicolumn{3}{|l|}{ Call triage } \\
\hline Critical & & $112(22.2)$ \\
\hline Semi Critical & & $392(77.6)$ \\
\hline \multicolumn{3}{|l|}{ Ambulance mechanism } \\
\hline Beacon (Light) & & $96(19.0)$ \\
\hline Siren & & $4(0.8)$ \\
\hline PA system & & $0(0.0)$ \\
\hline Light and siren & & $400(79.2)$ \\
\hline Light, siren \& PA system & & $5(1.0)$ \\
\hline \multicolumn{3}{|l|}{ Type of callero } \\
\hline MECC & & 337 (66.7) \\
\hline Public & & $138(27.3)$ \\
\hline Others $\mathrm{O}^{€}$ & & $29(5.7)$ \\
\hline \multicolumn{3}{|l|}{ Time call received ${ }^{\#}$} \\
\hline AM shift & & $170(33.7)$ \\
\hline PM shift & & $217(43.0)$ \\
\hline ON shift & & $118(23.4)$ \\
\hline Traveling time (minutes) & $7.97(4.54)$ & \\
\hline
\end{tabular}

*EMD Emergency Medical Dispatcher, SN Staff Nurse, AMO Assistant Medical Officer, APM Angkatan Pertahanan Malaysia (Malaysian Civil Defence personnel)

$£$ SPM is equivalent to $O$ level General Certificate of Secondary Education, STPM is equivalent to A level Higher School Certificate

$\infty$ MECC Medical Emergency Call Centre. For this study, MECC is located at main hospital of the city of Kota Bharu, Hospital Raja Perempuan Zainab II.

$€$ Others as the type of caller in this study refers to other emergency sector, clinic or private EMS within the coverage of HUSM ambulance service

\#AM shift: $7 a m$ to $2 \mathrm{pm}$, PM shift: $2 \mathrm{pm}$ to $9 \mathrm{pm}$, ON shift: $9 \mathrm{pm}$ to $7 \mathrm{am}$

Table 2: Mean Time of Ambulance Response (minutes)

\begin{tabular}{lcccc}
\hline Time & Mean & Min & Max & SD \\
\hline Call Duration & 2.11 & 0.00 & 7.00 & 1.25 \\
Team Activation & 2.33 & 0.00 & 25.00 & 1.95 \\
Travelling Time (GIS) & 7.98 & 1.00 & 27.01 & 4.54 \\
Ambulance Response Time & 12.42 & 3.00 & 44.00 & 5.39 \\
\hline
\end{tabular}


Table 3: Univariate analysis of the association between internal factor and ambulance response time

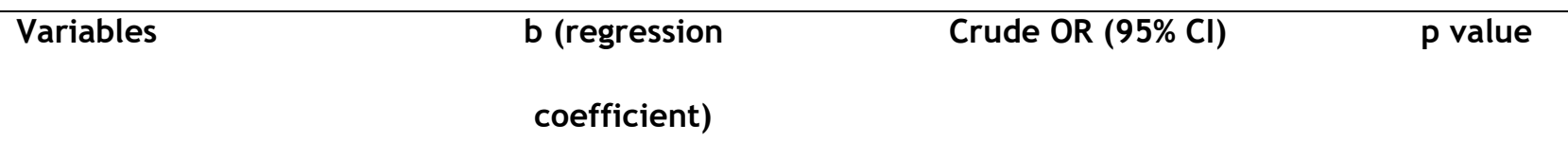

\section{Call receiver}

EMD

0

1

SN/AMO

0.70

$2.02(0.76,5.34)$

0.159

APM

$-0.26$

$0.77(0.42,1.41)$

0.398

Highest Academic

Qualification

SPM/STPM

0

1

Diploma

0.61

$1.85(0.69,4.95)$

0.223

Degree

20.25

$621336489.92(0.00,0.00)$

$>0.950$

Working Experience

0.012

$1.01(0.98,1.05)$

0.459

Caller type

Public

MECC

0

1

Others

$-0.98$

$0.38(0.24,0.59)$

$<0.001$

$-1.86$

$0.16(0.07,0.34)$

$<0.001$

Call duration

0.52

$1.67(1.37,2.05)$

$<0.001$

There are a few limitations faced while conducting this study such as sample bias. We only included the responded case which used ambulances that were installed with AVL- GIS system. Hence, in this study the sample size is smaller than the exact number of responded case. There was also bias in the continuity of data as the call receiver and the person who went for ambulance calls were different persons. This caused differences in time recall during form filling. Few measures had been taken care to avoid this issue. The call receiver would fill up the form regarding the call details before handed it over to the response team for continuity of data entry.
Occasionally, there is unavailability of the ambulance service which is supposed to be under the coverage of primary healthcare service of that area at particular times. There is only one ambulance available at each healthcare clinic in Kota Bharu and when the ambulance is in service or is used to respond to another case, our ambulance will be called for response. Another reason is that during mass incident, our ambulance will also be asked to respond to the area outside their coverage in order to assist in providing emergency care on site and for transportation of patients involved to nearby healthcare centre. This issue might lead to a delayed response time due to longer distance than the usual coverage area. 
Table 4: Univariate analysis of the association between external factors and ambulance response time

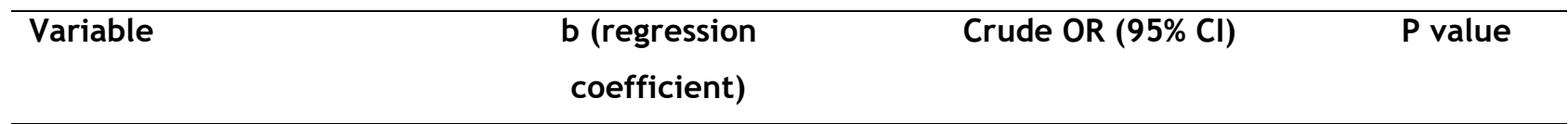

\section{Call triage}

Critical

0

Semi critical

$-0.57$

1

Team allocation time

0.45

$0.56(0.33,0.96)$

0.035

Team allocation time

\section{Response location}

Inside Building

0

$1.57(1.32,1.85)$

$<0.001$

Outside Building

$-0.04$

1

0.843

Traffic condition to scene

Non heavy

0

$0.96(0.63,1.45)$

Heavy

$-0.76$

$0.47(0.25,0.90)$

0.023

\section{Ambulance Mechanism}

Siren

0

1

Light (beacon)

0.85

$2.33(0.23,23.25)$

0.470

Siren and light

1.06

$2.88(1.80,4.61)$

$<0.001$

Siren, light and PA System

1.14

$3.11(0.34,28.88)$

0.318

\section{Patient contact time}

0.002

$1.00(0.95,1.06)$

0.950

\section{Weather}

Good

0

1

Bad

0.52

$1.68(0.79,3.55)$

0.177

Time call taken

AM shift

0

1

PM shift

0.25

$1.29(0.82,2.03)$

0.280

ON shift

0.26

$1.30(0.76,2.23)$

0.337

Travelling time

1.31

$3.7(2.82,4.85)$

$<0.001$

${ }^{*}$ The significant external factors associated with delayed ambulance response time $(p<0.05)$ are traffic condition, team allocation time and travelling time.

\#Ambulance mechanism used in delayed response case is significantly affected whereas siren and light 2.88 time higher used compare to siren alone. 
Table 5: Multivariate analysis of the association between internal and external factors towards delayed ambulance response time

\begin{tabular}{llcc}
\hline Variable & $\begin{array}{l}\text { b (regression } \\
\text { coefficient) }\end{array}$ & Adjusted OR (95\% Cl) & P value \\
\hline Team Allocation Time & 1.48 & $4.41(2.73,7.12)$ & $<0.001$ \\
Travelling Time (GIS) & 1.85 & $6.33(4.06,9.88)$ & $<0.001$ \\
\hline
\end{tabular}

\section{CONCLUSION}

This study found that call duration, caller type, traffic condition, travelling time and team allocation time were factor associated with delayed ambulance response time. Further evaluation on each factor is needed and intervention should be made towards these problems as to prevent delayed response time and improve the prehospital service in the future.

\section{Conflict of interest}

The authors declare no potential conflict of interest.

\section{Acknowledgements}

This study has been funded by Universiti Sains Malaysia (USM) short term grant (304/PPSP/61313191).

\section{REFERENCES}

1. Eisenberg MS, Bergner L, Hallstrom A. Cardiac Resuscitation in The Community. Importance of Rapid Provision and Implications for Program Planning. JAMA.1979; 241(18):19051907.

2. Shah $\mathrm{CH}$, Ismail IM, Mohsin SS. Ambulance Response Time and Emergency Medical Dispatcher Program: A Study in Kelantan, Malaysia. Southeast Asian J Trop Med Public Health. 2008; 39(6):1150-1154.

3. Do YK, Foo $\mathrm{K}$, Ng YY, Ong MEH. A Quantile Regression Analysis of Ambulance Response Time. Prehospital Emergency Care. 2013;17(2):170-176.

4. Breen N, Woods J, Bury G, Murphy AW, Brazier H. A National Census of Ambulance Response Times to Emergency Calls in Ireland. J Accid Emerg Med. 2000;17(6):392-395.

5. Chin SN, Cheah PK, Arifin MY, Wong BL, Omar Z, Yassin FM, Gabda D. Determinants of Ambulance Response Time: A Study in Sabah, Malaysia. Proceedings from AIP Conference
Proceedings. 2017

6. Lam SSW, Nguyen FNHL., Ng YY, Lee VPX, Wong TH, Fook C, Ong MEH. Factors Affecting the Ambulance Response Times of Trauma Incidents in Singapore. Accident Analysis \& Prevention. 2015;82:27-35.

7. McMeekin P, Gray J, Ford GA, Duckett J, Price Cl. A Comparison of Actual Versus Predicted Emergency Ambulance Journey Times Using Generic Geographic Information System Software. Emergency Medicine Journal. 2014;31(9):758-762.

8. Campbell JP, Gratton MC, Salomone JA, Watson WA. Ambulance Arrival to Patient Contact: The Hidden Component of Prehospital Response Time Intervals. Annals of Emergency Medicine. 1993;22(8):1254-1257.

9. Morrison LJ, Angelini MP, Vermeulen MJ, Schwartz B. Measuring the EMS Patient Access Time Interval and The Impact of Responding to High-Rise Buildings. Prehospital Emergency Care. 2005;9(1):14-18.

10. Mohd Uzir M. Current Population Estimates, Malaysia, 2017-2018. Official Portal, Department of Statistics Malaysia. 2018

11. Carr BG, Caplan JM, Pryor JP, Branas CC. A Meta-Analysis of Prehospital Care Times for Trauma. Prehospital Emergency Care. 2006;10(2):198-206.

12. Peduzzi P, Concato J, Kemper E, Holford TR, Feinstein AR. A simulation study of the number of events per variable in logistic regression analysis. Journal of clinical epidemiology. 1996 Dec 1;49(12):1373-1379. 